\title{
BMJ Open Reconnecting mothers and children after violence (RECOVER): a feasibility study protocol of child-parent psychotherapy in Australia
}

\author{
Leesa Hooker, ${ }^{\oplus 1,2}$ Emma Toone, ${ }^{1,3}$ Vibhay Raykar, ${ }^{4}$ Cathy Humphreys, ${ }^{5}$ \\ Anita Morris, ${ }^{06}$ Elizabeth Westrupp, ${ }^{1,7}$ Angela Taft ${ }^{1}$
}

To cite: Hooker L, Toone E, Raykar V, et al. Reconnecting mothers and children after violence (RECOVER): a feasibility study protocol of child-parent psychotherapy in Australia. BMJ Open 2019;9:e023653. doi:10.1136/ bmjopen-2018-023653

- Prepublication history for this paper is available online To view these files, please visit the journal online (http://dx.doi. org/10.1136/bmjopen-2018023653).

Received 17 April 2018 Revised 18 January 2019 Accepted 15 February 2019

Check for updates

(C) Author(s) (or their employer(s)) 2019. Re-use permitted under CC BY-NC. No commercial re-use. See rights and permissions. Published by BMJ.

For numbered affiliations see end of article.

Correspondence to

Dr Leesa Hooker;

I.hooker@latrobe.edu.au

\section{ABSTRACT}

Introduction Intimate partner violence detrimentally affects the social and emotional well-being of children and mothers. These two populations are impacted both individually and within the context of their relationship with one another. Child mental health, maternal mental health and the mother-child relationship may be impaired as a consequence. Early intervention to prevent or arrest impaired mother-child attachment and child development is needed. Dyadic or relational mental health interventions that include mothers with their children, such as childparent psychotherapy, are effective in improving the mental health of both children and mothers and also strengthening their relationship. While child-parent psychotherapy has been trialled overseas in several populations, Australian research on relational interventions for children and women recovering from violence is limited. This study aims to assess the acceptability and feasibility of implementing child-parent psychotherapy in Australian families.

Methods and analysis Using a mixed methods, prepost design this feasibility study will examine the acceptability of the intervention to women with preschool aged children ( $3-5$ years, $n=15$ dyads) and providers, and identify process issues including recruitment, retention and barriers to implementation and sustainability. In addition, intervention efficacy will be assessed using maternal and child health outcomes and functioning, and mother-child attachment measures. Young children's mental health needs are underserviced in Australia. More research is needed to fully understand parenting in the context of intimate partner violence and what works to help women and children recover. If the intervention is found to be feasible, findings will inform future trials and expansion of child-parent psychotherapy in Australia.

Ethics and dissemination Ethics approval obtained from clinical sites and the La Trobe University Human Research Ethics Committee (ID: HEC17-108). Results will be disseminated through conference proceedings and academic publications.

\section{INTRODUCTION}

Intimate partner violence

Although there are many forms of violence that occur within parental couples and
Strengths and limitations of this study

- Strengths of this study include contributions from multiple sources (ie, mothers, clinicians and managers) and the use of independent observations and interviews.

- The use of implementation theory provides a strong conceptual base to explore contextual factors that impact on model integration.

- These contextual factors will be of international relevance to other researchers exploring the use of child-parent psychotherapy.

- Limitations include the sample size, lack of a comparison arm and follow-up postintervention which reflects the emphasis on feasibility rather than efficacy within the Australian context.

towards children, ${ }^{1}$ intimate partner violence (IPV) is overwhelmingly a gendered form of violence that specifically effects both child and mother simultaneously. ${ }^{2}$ IPV is defined as behaviour by a partner or ex-partner that includes physical, sexual and/or psychological abuse and controlling behaviours. This violence results in significant social, health and economic costs. ${ }^{23}$ One in three women have experienced some form of IPV in their lifetime. ${ }^{2}$ IPV is more prevalent in women of childbearing age (especially with children under 5$)^{4}$ and consequently, children are frequently exposed and affected by the abuse. These children are also at greater risk of experiencing other forms of child abuse. ${ }^{5} \mathrm{Up}$ to 1.9 million (or $43 \%$ ) of Australian children witness IPV and/or parental conflict. ${ }^{6}$

IPV and children

Early life exposure to abuse and trauma is harmful to the growing brain, with profound cognitive and developmental effects. IPV can be defined as a traumatic experience and can lead to poor mental health and diagnostic problems such as posttraumatic stress 
disorder (PTSD). High cortisol levels associated with witnessing or experiencing IPV can impact on children's immune function, with persistent fear and anxiety activating a prolonged stress response, which is toxic and changes the structure and physiology of the child's developing brain. ${ }^{7}$ This is especially so in the areas of the brain associated with emotions and learning, where changes can in turn impact on behaviour, controlling and focussing attention, memory and decision making. ${ }^{7}$

The first 5 years of life represent a critical developmental period making infants and young children particularly vulnerable to the harmful and pervasive developmental impacts of IPV. ${ }^{4}$ Consequently, children exposed to IPV may suffer a range of serious sociobehavioural, academic, mental and physical health problems. Meta-analyses show that the most common child mental health conditions associated with IPV include mood disorders, behaviour problems and trauma symptoms. ${ }^{89}$

Adverse childhood experiences, which include exposure to IPV and child abuse, are associated with lifelong negative consequences, including physical and mental health issues, substance use, long-term chronic illness into adulthood and premature death. ${ }^{10} \mathrm{~A}$ dose response is also evident, as the greater the exposure to adversity (duration, intensity and cumulative harm), the greater the subsequent impairment. ${ }^{3}$ The co-occurrence of IPV and child abuse also puts children at further risk of victimisation and ongoing intergenerational violence in later life. $^{5}$

Infant mental disorders contribute towards a significant disease burden worldwide. ${ }^{4}$ Intervening to support young children in their caregiving relationships after violence can decrease their immediate suffering and address potential future mental health risk. ${ }^{41}$ Consequently, more government investment in prevention and early intervention would be cost effective and is urgently needed. ${ }^{312}$

\section{IPV and mothering}

Parenting in the context of IPV is a complex and under-researched area. ${ }^{13}$ IPV may have a negative impact on women's parenting capacity by reducing their emotional and physical availability and competence to attend to their children's needs. ${ }^{13-15}$ In addition to the direct abuse, the person using violence may employ deliberate strategies to undermine women's parenting, including involving children in dynamics associated with the coercion and control of their mother. ${ }^{16}$ The person using violence will often denigrate her social expectations and identity as a 'good mother' ${ }^{17}$ Mothers may also denigrate and blame themselves for failing to protect their children from violence. ${ }^{18}$ These abusive dynamics interfere with motherhood, ${ }^{19}$ often depriving women and children of a mutually enjoyable relationship with one another. ${ }^{16}$ Violence in the home is often not discussed between mothers and children, which detrimentally affects the individual and dyadic relationship. ${ }^{16}$

\section{Resiliency and attachment}

The consequences of IPV (for the child) vary according to individual, family and community resiliency. ${ }^{5}$ Not everyone who experiences a traumatic event also experiences a mental health disorder, but some do become symptomatic and need assistance. Supportive grandparents, positive friendship networks and positive peer or sibling relationships are protective against the negative consequences of abuse. Self-esteem and a sense of control or self-efficacy are core building blocks of resilience. ${ }^{5}$ Love, consistent and supportive care from the non-violent parent are important factors in mitigating child trauma. ${ }^{20}$ Mother-child dyads that display secure attachment relationships (where the child feels their parent consistently meets their emotional and physical needs) are associated with children having fewer behavioural problems, higher levels of social competence, language skills and school readiness and being less reliant on adults, compared with insecure children. ${ }^{21}$ While there is extensive evidence that exposure to IPV is associated with serious physical and mental health issues, very few therapeutic relational interventions exist to address the violence and trauma experienced by women and children, and to heal the mother-child relationship. ${ }^{13}$

\section{Interventions for children}

While accurate data on the prevalence and burden of mental health disorders in infants and young children is difficult to determine (poorly reported and poorly identified), global evidence suggests up to $20 \%$ of young children ( $0-5$ years) suffer mental health disorders, similar rates to that of older children and adolescents. ${ }^{4}$ Young children's mental health needs are significantly underserviced in Australia, with less than $1 \%$ of $0-4$ year olds receiving a mental health service. ${ }^{12}$ Interventions for children exposed to IPV range from crisis, counselling and/or group therapeutic work, and multicomponent interventions combining parenting, advocacy, psychoeducation, child mental health and social support. Programme efficacy is difficult to determine as few programmes are evidence based or extensively evaluated. ${ }^{22}$

Recent review evidence suggests psychotherapeutic and psychoeducational programmes focused on behavioural and mental health outcomes are associated with modest improvements, although study quality is poor and the sustainability of effects beyond 1 month is unknown. ${ }^{23}{ }^{24}$ There have been calls for IPV intervention outcome measures to be expanded beyond child health to explore meaningful outcomes for parents, child participants and practitioners. ${ }^{25}$ Beyond trauma symptoms, the maternal health and well-being benefits of dyad therapy for women affected by IPV are yet to be determined. More high-quality trials are needed to evaluate the effectiveness of psychological interventions for children exposed to trauma. ${ }^{24}$ Researchers working together on existing programmes (rather than developing new ones) can enhance scholarship in the field, 
with consensus on a standardised outcome set that includes more functional outcomes. ${ }^{2325}$

\section{Child-parent psychotherapy}

The strongest evidence from reviews include international randomised controlled trials ${ }^{2627}$ which highlight the therapeutic benefit of working with mothers and children together in the aftermath of IPV. Psychotherapeutic interventions (such as child-parent psychotherapy $[\mathrm{CPP}]$ ) that include combined sessions with the mother and child, show significant and positive differences for the mental health of mothers and the behavioural and emotional functioning of children, compared with controls. ${ }^{27}$ Dyadic interventions with a focus on maternal sensitivity and reflective function (ie, mothers' ability to reflect on her own and her child's behaviour, thoughts and feelings; and on her personal experience and history affecting current caregiving interactions) show most benefit. ${ }^{28}$

CPP is a relationship-based treatment for parent-child dyads (children from birth to 5 years) who have been traumatised by violence and are experiencing emotional, attachment and behavioural difficulties. The treatment is based on various theories including attachment, parenting, trauma, social learning, cognitive behavioural theories and intergenerational transmission of child maltreatment and violence. ${ }^{29} \mathrm{CPP}$ has been evaluated in several US-based randomised controlled trials with IPV exposed and maltreated children, ${ }^{27}{ }^{30-32}$ with at risk samples such as anxiously attached dyads ${ }^{11}$ and depressed mothers and their children. ${ }^{33-35}$

The main goals of CPP are to reduce child trauma symptoms and behavioural problems, and to strengthen the relationship between the mother and the child exposed to IPV. The dyad relationship acts as the vehicle for restoring the child's normal developmental trajectory, including children's general functioning and sense of safety and their primary attachment relationships. The cocreation of trauma narratives facilitate healing and resolution. ${ }^{29}$ Combined psychotherapy for children exposed to IPV is now recommended by the $\mathrm{WHO},{ }^{36}$ consequently testing of CPP has expanded beyond the USA. ${ }^{37} 38$

\section{Access to evidence-based family violence and trauma informed relational therapy in Australia}

Therapeutic services are available to Australian women and children who have experienced trauma, often through counselling and mental health services. However, services including mental health, child welfare and family violence are often siloed, with separate services for women and children. ${ }^{39}$

Where there is ongoing, postseparation IPV and symptoms of trauma (that are subthreshold for a mental health diagnosis), child and adolescent mental health services may refer clients to either child protection or to a primary or secondary healthcare service. If mothers are assessed as being 'protective' (not perpetrating the violence), the family becomes ineligible for specialised therapeutic services. Healthcare practitioners may or may not be trained in providing evidence-based dyadic relational intervention or family violence risk assessment. Family violence services may or may not employ trained infant and child mental health professionals or have clinical governance processes in place to support quality child interventions. This leaves a significant population of mother and child victims unable to access recovery options. ${ }^{40}$ Subsequently, very few Australian services have offered dyadic therapy for IPV victims, ${ }^{13}{ }^{40}$ nor have programmes been rigorously evaluated. ${ }^{1341}$

Although dyadic/triadic interventions for the whole family, including fathers (as parents and previous users of violence) are required, ${ }^{42}$ in this feasibility study, we are only focusing on mother-child dyads (like the original Lieberman trial) ${ }^{27}$ to explore improvements in the mother-child relationship. Considering the strong level of evidence on the consequences of IPV, the effectiveness of CPP and dyad therapies for children exposed and the call for more intervention research in the field, the Reconnecting mothers and children after violence (RECOVER) project team proposes to implement and evaluate the Lieberman $e t a l,{ }^{29} \mathrm{CPP}$ model in Australia.

The protocol and feasibility study findings will assist international researchers investigating CPP, and if effective, inform the implementation of a future randomised controlled trial and facilitate wider implementation of the treatment programme throughout Australia.

\section{AIM AND RESEARCH QUESTIONS}

To implement and evaluate the CPP model ${ }^{29}$ of care for Australian families.

The RECOVER project will address the following research questions:

1. How acceptable and feasible is CPP to the Australian context?

a. How acceptable is the intervention (and its evaluation) for women and service providers?

b. What are the barriers and facilitators to implementation of the CPP model into services?

c. What (if any) are the dyad recruitment and retention issues encountered?

d. Are there process and impact differences between service providers in metropolitan and rural intervention sites?

2. How closely do trainee therapists adhere to CPP model fidelity?

a. How well do therapists undergoing CPP training and delivering CPP, adhere to CPP programme goals and model fidelity at each dyad session?

3. How effective is treatment during the study?

a. Do women's and children's trauma symptoms differ before and after treatment?

b. Does treatment improve the mother-child relationship? 


\section{METHODS AND ANALYSIS \\ Design}

We propose a concurrent, mixed methods feasibility study to assess acceptability, implementation processes and test model efficacy. In a longitudinal, repeated measure design, reliable outcome measures will be assessed at three time points: baseline (preintervention), 12 weeks into the core CPP phase (midpoint) and at the completion of the intervention (post). Clinical data of measures will be collected from service records. Qualitative indepth interviews with therapists and managers will occur mid-intervention and postintervention. Women will be interviewed post-treatment. Direct observation of dyad interaction throughout the treatment will be recorded and assessed.

Therapeutic interventions to support women and children need to be sustainable. Process evaluation will help us to understand the feasibility of implementation and sustainability. In this study, factors influencing integration of the CPP model will be explored using May and Finch's implementation framework-normalisation process theory (NPT). ${ }^{43}$

\section{Participants}

We aim to recruit 15 dyads from specialist, domestic violence and child and adolescent mental health services via intake systems. These study sites have been chosen due to interest from service providers and existing partnerships. As CPP training takes 18 months to complete, all therapists will be undergoing CPP training during the time of the study. Masters level clinicians (social workers, mental health nurses, child psychotherapists and psychologists), with experience in infant-parent mental health and currently working in the above clinical settings, were invited to complete CPP training and deliver the intervention.

The CPP training and supervision is conducted by an endorsed international CPP trainer from the USA. In this study, the small sample reflects the emphasis on exploring feasibility rather than efficacy.

\section{Dyad recruitment criteria}

\section{Inclusion criteria}

- Preschool aged child (3-5 years).

- Biological mother of child.

- English speaking mother.

- Recent exposure to IPV (past 12 months).

- Mother has received subsequent IPV assessment and advocacy support (risk assessment and safety plan, counselling and referral to legal, housing, healthcare).

- Postcrisis or not living with the person using violence.

- Maternal readiness to engage

- Clinical signs of trauma (eg, child presenting with emotional or behavioural issues/impaired motherchild interaction based on clinical assessment).

\section{Exclusion criteria}

- Mother-child dyads where the person using violence continues to use violence and live in the family home or there is insufficient safety.
- Mothers who have been substantiated/documented abusers of the child.

- Mothers who are currently abusing substances.

- Previous diagnosis of maternal intellectual disability.

- Significant maternal mental illness, for example, psychosis.

- Children with a significant intellectual disability and/ or autism which interferes with their capacity for engagement in treatment.

\section{Recruitment process}

The majority of participants will be recruited and receive treatment through domestic violence and child and adolescent mental health services, in metropolitan and regional areas. Due to organisational capacity, a smaller number of dyads will be recruited from other services such as maternal and child health. Potential motherchild dyads entering services will be screened by CPP therapists to determine participant eligibility for the project, describe the study and gain informed consent.

\section{Intervention}

CPP is a psychotherapeutic intervention for parents and children who have experienced some form of trauma. Delivered in hour long weekly sessions, by specialist therapists, core treatment ranges from 20 to 32 weeks or more, depending on family need, ${ }^{29}$ and includes three phases:

1. Foundation phase-assessment and engagement sessions (4-6 weeks).

2. Core intervention length varies depending on the need.

3. Termination-promoting sustainability and post assessment $(<8$ weeks $) .{ }^{29}$

The type of trauma exposure, child age, developmental status and clinical symptoms all determine the structure and length of the CPP sessions. Therapy for young infants and children includes working with the mother to help her understand how the experience has impacted on the child's functioning. Play-based therapy is used with older children to facilitate communication between the mother and child. The therapist enables past maternal trauma to be acknowledged, including how this trauma can interfere with the mother's response to the child and alternative, more developmentally appropriate ways of interacting with her child are addressed. ${ }^{29}$ Ongoing risk and safety assessment/planning will be completed by therapists at regular intervals during treatment and appropriate referral made to supports when needed. Maternal survey questions also ask about ongoing abuse and exposure to the perpetrator in the past month.

\section{CPP providers and training}

Through training, peer supervision and clinical practice opportunities, this project will develop and promote a national CPP Community of Practice that aims to build workforce capacity, enhance practice, and provide a vehicle for ongoing education and professional networking for those working in the field. Currently, 
there is a lack of professionally qualified therapists or clinicians with specialist training in family violence and trauma informed care, and evidence-based relational therapy. ${ }^{40}$ The project will work to redress this gap, as part of the broader emerging family violence therapeutic service system reforms in Victoria, by building an experienced, specialised workforce in CPP and trauma and violence informed, mental healthcare for young children and their mothers.

\section{Process evaluation \\ Implementation}

We will use NPT to design the evaluation and explore processes of implementation of the CPP model. ${ }^{43}$ This sociobehavioural theory provides a conceptual framework for understanding 'the social organisation of the work (implementation), of making practices routine elements in everyday life (embedding), and of sustaining embedded practices in their social contexts (integration) ' ${ }^{43}$ NPT consists of four core inter-related working principles needed for successful integration of new practices-coherence (sense making work), cognitive participation (relational work), collective action (operational work) and reflexive monitoring (appraisal work). These principles will frame the data collection tools and support the intervention and interpretation/evaluation processes, helping to pragmatically explain the dynamics of how the CPP model is implemented. In addition to qualitative process evaluation, descriptive quantitative data on intervention fidelity, reach and dose will be collected.

\section{Intervention fidelity, reach and dose}

CPP fidelity monitoring is essential due to potential diversity of clinical presentations and programme flexibility. The CPP manual describes a six strand fidelity framework, which includes reflective practice, emotional process, dyadic relations, trauma framework, procedural and content fidelity. ${ }^{29}$ Each CPP phase has detailed fidelity tools incorporating this framework, which will be completed by clinicians. Clinicians will complete fidelity logbooks after each consultation, which indicate adherence to CPP goals. Throughout official CPP training, treatment fidelity is closely monitored through review of process notes and regular clinical supervision. Clinical data will be collected on maternal, child and/or dyad assessment, fidelity measures, and the intervention reach (number of eligible dyads offered CPP) and dose (sessions attended/delivered).

\section{Data management}

Participants and therapists will be given a unique ID code for use throughout the study. A secure, purposebuilt online electronic database will be used to record and store all data including participant and therapist details. Dyadic interaction will be recorded using tablet devices. Onsite clinical researchers will regularly collate intake, fidelity and survey data. All data will be uploaded as electronic files to the university research drives that are securely stored.

\section{Outcome measures}

When deciding on outcomes measures to use in this study, we considered (a) measures used in the original Lieberman trial $^{27}$ and those recommended by CPP developers; (b) calls within the literature to expand assessment beyond child mental health and behaviour; (c) participant and therapist burden; (d) service provider capacity and experience; (e) instrument and training costs and (f) the age of children.

Primary outcomes include maternal and child mental health and trauma (PTSD) symptoms and child behavioural problems. PTSD in 3-5-year olds is often displayed as behaviour problems and these are used as a basis for description of the disorder (table 1).

Secondary outcomes will measure recovery by assessing parenting (self-efficacy, warmth, irritability and consistency), reflective functioning (or the mother's ability to hold the child's mental states in mind), responsiveness and the mother-child relationship/interaction.

\section{Data collection time points \\ Preintervention}

Therapists will collect baseline quantitative outcome measures using a maternal survey (and parent-child interaction free play recording) in the foundation phase of treatment. Survey responses will also capture women's expectations of the treatment and outcomes that most suit their needs. Further details on outcome measures are provided in table 1 . Therapists will document fidelity, attendance rates and dose for each dyad at each session.

\section{Midpoint}

While the true midpoint of a flexible, needs based intervention is undetermined, findings from previous CPP studies suggest dyads require between 20 and 32 weeks of core therapy. Consequently, we have defined our midpoint evaluation to be at 12 weeks. Researchers will conduct process evaluation interviews with CPP therapists and managers using NPT, to explore feasibility, experiences and contextual factors related to implementation. Therapists will also collect maternal report of quantitative outcome measures and record direct observation of dyad free play.

\section{Postintervention}

Researchers will conduct impact evaluation interviews with mothers, therapists and managers using NPT, to explore feasibility, perceptions/experiences and contextual factors related to implementation. Quantitative outcome measures and dyad free play recording will be completed by therapists in the termination phase of treatment.

\section{Analysis}

Interview data will be audio recorded, transcribed, coded and analysed using an NPT lens. Process 
Table 1 RECOVER project outcome measures and data collection time points

\begin{tabular}{|c|c|c|c|}
\hline Outcome measure & Pre & Mid & Post \\
\hline \multicolumn{4}{|l|}{ Maternal health and well-being } \\
\hline Symptoms Checklist-90 Revised ${ }^{49}$ & $x$ & $x$ & $x$ \\
\hline PTSD Symptom Scale-Interview ${ }^{50}$ & $x$ & $\mathrm{x}$ & $\mathrm{x}$ \\
\hline Composite Abuse Scale ${ }^{51}$ & $x$ & & $x$ \\
\hline \multicolumn{4}{|l|}{ Child functioning and trauma symptoms } \\
\hline Strengths and Difficulties Questionnaire ${ }^{52}$ & $\mathrm{x}$ & $\mathrm{x}$ & $\mathrm{x}$ \\
\hline Young Child PTSD Checklist ${ }^{53}$ & $\mathrm{x}$ & $\mathrm{x}$ & $\mathrm{x}$ \\
\hline \multicolumn{4}{|l|}{ Parenting } \\
\hline Parental self-efficacy, warmth, irritability and consistency ${ }^{54}$ & $x$ & $\mathrm{x}$ & $\mathrm{x}$ \\
\hline \multicolumn{4}{|l|}{ Mother-child relationship } \\
\hline Parental Reflective Functioning Questionnaire ${ }^{55}$ & $x$ & $\mathrm{x}$ & $\mathrm{x}$ \\
\hline $\begin{array}{l}\text { Recording of parent-child interaction assessed using } \\
\text { Coding of Attachment-Related Parenting }\end{array}$ & $\mathrm{x}$ & $\mathrm{x}$ & $\mathrm{x}$ \\
\hline
\end{tabular}

PTSD, posttraumatic stress disorder.

evaluation will assess the model's feasibility and identify contextual programme barriers and facilitators to inform future research. NVivo ${ }^{44}$ coding software will be used to assist the analysis, and a portion cross-coded for reliability. Descriptive and inferential statistical analyses will be completed using STATA V.15. ${ }^{45}$ Proportions and means will be calculated to describe fidelity and feasibility measures. Paired-samples t-tests will compare mean scores at different time points. Effect size calculations will detect meaningful change in outcomes across time, among this small sample. Comparison will also be made between rural/regional and metropolitan groups and with the findings from the original Lieberman trial and follow-up study. ${ }^{27}{ }^{30}$ Direct observation data (video recordings) will be viewed and coded using a coding scheme specifically designed for observation of parentchild interaction called Coding of Attachment-Related Parenting (CARP) ${ }^{46}$ Domains include parental responsiveness, sensitivity, affect, child affect and dyad mutuality. Ten percent of recordings will be double-coded. If parents do not consent to recording, then therapists will make notes of parent-child interaction and these notes will then be coded and analysed using the CARP guide.

\section{Cost evaluation}

A cost-benefit analysis is unable to be determined in this prepost study design due to the lack of a comparison group. In preparation and to inform our future randomised controlled trial, we plan to assess the feasibility and acceptability of capturing the cost of intervention data. Postintervention, costs such as therapist training and provider delivery (salaries and travel) will be collected, along with the costs to women attending sessions (travel to therapy and child care for older children).

\section{Patient and public involvement}

This paper is a protocol outlining the process of assessing the feasibility of implementing CPP into the Australian context. There was no patient or public involvement in establishing the research questions or study design; however, participant burden was considered in the design and data collection methods, and patient experience will be explored as part of the feasibility and acceptability of the programme. Study findings will be available to study participants enrolled in the study, with dissemination of findings to service organisations.

\section{DISCUSSION}

There is an urgent need for effective therapeutic treatment options for women and children exposed to violence. While the evidence base regarding the acceptability, effectiveness and costs of interventions is unclear, ${ }^{23}$ the US-based Lieberman $e t a l^{29} \mathrm{CPP}$ model of treatment appears to be most effective for mother-child dyads exposed to violence. In Australia, while treatment options do exist for women and children, programmes have historically been delivered by poorly funded, community organisations and as such, have not been rigorously evaluated. ${ }^{13}$

This study aims to assess the feasibility of introducing the Liebermann et $a l^{29}$ model of CPP to Australian families, to enable healing from trauma and repair of the mother-child relationship after IPV. A sound feasibility study is required to test implementation of the CPP model in the Australian context, including how the intervention may be adapted for delivery in community-based urban, regional and rural settings.

As has been seen with other effective complex interventions, adaptations into other countries may not show similar results. ${ }^{47}$ Australian healthcare, service delivery 
and family needs are very different to the USA (funded health services, differences in population diversity/ culture) and need to be considered when implementing CPP. Lynch ${ }^{48}$ cautions that 'positive effects found in studies conducted decades ago and in other countries where control conditions or usual care are vastly different to those in 21st-century Australia ought to be carefully scrutinised'. ${ }^{48}$

More Australian research is needed to fully understand parenting in the context of abuse and what works to help women and children recover. Dyadic psychotherapeutic interventions may be most effective given that the CPP methodology offers an early intervention pathway to mitigate the long-term effects of IPV on families and developing children. If feasible and effective, findings will inform future trialling and expansion of CPP nationally.

\section{Study timeline}

The project started in January 2017, with expert reference group/steering committee input monthly during the 12-month planning phase. Applications for ethical approval were submitted in November 2017 and January 2018, with approval received in December 2017 and February 2018. This study began recruiting in February (domestic violence services) and March (child mental health service) 2018 with plans for a 3-month recruitment phase. Intervention will be up to 12 months, with completion of the project scheduled for January 2020.

\section{Author affiliations}

${ }^{1} J u d i t h$ Lumley Centre, School of Nursing and Midwifery, La Trobe University, Bundoora, Victoria, Australia

${ }^{2}$ Rural Department of Nursing and Midwifery, La Trobe Rural Health School, Bendigo, Victoria, Australia

${ }^{3}$ Berry Street Childhood Institute, Richmond, Victoria, Australia

${ }^{4}$ Child and Adolescent Mental Health, Goulburn Valley Health, Shepparton, Victoria,

Australia

${ }^{5}$ Department of Social Work, The University of Melbourne, Melbourne, Victoria, Australia

${ }^{6}$ Office of Professional Practice, Department of Health and Human Services, Victorian Government, Melbourne, Victoria, Australia

${ }^{7}$ School of Psychology, Deakin University, Melbourne Burwood Campus, Burwood, Victoria, Australia

Acknowledgements We would like to thank our US colleagues, Professor Chandra Ghosh-Ippen (CPP cofounder) and Professor Julie Larrieu (CPP trainer) for their input into the design of the study and introductions to international colleagues from Sweden and Norway. Thanks to Swedish colleagues for sharing their knowledge of CPP training and feasibility. We would also like to acknowledge the Berry Street Childhood Institute for bringing the CPP training to Melbourne, and in particular, Marg Hamley for all her project support.

Contributors $\mathrm{CH}$ and AT are chief investigators for this study. $\mathrm{LH}$ is a project coordinator, who drafted the manuscript, ethics submissions and document preparation. All authors (LH, ET, VR, CH, EW, AM and AT) contributed to study design. $E T$ and VR are site clinicians providing valuable input on practice considerations and organisational integration of the intervention. All authors reviewed and approved the final version of this manuscript.

Funding This work was supported by the Australian National Health and Medical Research Council (NHMRC) Centres for Research Excellence Grant (GNT 1116690) and Berry Street Childhood Institute. Dr Westrupp was supported by Australian Communities Foundation through the Roberta Holmes Transition to Contemporary Parenthood Program at La Trobe University (Coronella sub-fund). Clinical research partners also funded the extensive CPP training for all therapists involved in the study.

Competing interests None declared.

Patient consent for publication Not required.

Ethics approval Ethics approval was obtained from all clinical sites and the La Trobe University Human Research Ethics Committee also approved the project (ID: HEC17-108). On completion, results of the study will be published in academic journals and presented at national and international conferences.

Provenance and peer review Not commissioned; externally peer reviewed.

Open access This is an open access article distributed in accordance with the Creative Commons Attribution Non Commercial (CC BY-NC 4.0) license, which permits others to distribute, remix, adapt, build upon this work non-commercially, and license their derivative works on different terms, provided the original work is properly cited, appropriate credit is given, any changes made indicated, and the use is non-commercial. See: http://creativecommons.org/licenses/by-nc/4.0/.

\section{REFERENCES}

1. Kelly JB, Johnson MP. Differentiation among types of intimate partner violence: research update and implications for interventions Family Court Review. 2008;46:476-99.

2. World Health Organization. Global and regional estimates of violence against women: prevalence and health effects of intimate partner violence and non partner sexual violence: World Health Organization, 2013.

3. Rivara FP, Anderson ML, Fishman P, et al. Intimate partner violence and health care costs and utilization for children living in the home. Pediatrics 2007;120:1270-7.

4. Lyons-Ruth K, Todd Manly J, Von Klitzing K, et al. The worldwide burden of infant mental and emotional disorder: report of the task force of the World Association for infant mental health. Infant Ment Health J 2017;38:695-705.

5. Holt S, Buckley H, Whelan S. The impact of exposure to domestic violence on children and young people: a review of the literature. Child Abuse Negl 2008;32:797-810.

6. Westrupp EM, Rose N, Nicholson JM, et al. Exposure to interparental conflict across 10 years of childhood: data from the longitudinal study of Australian Children. Matern Child Health $J$ 2015;19:1966-73.

7. National Scientific Council on the Developing Child. Persistent Fear and Anxiety Can Affect Young Children's Learning and Development: Working Paper. 2010 http://developingchild.harvard.edu/resources/ persistent-fear-and-anxiety-can-affect-young-childrens-learningand-development/

8. Evans SE, Davies C, DiLillo D. Exposure to domestic violence: a meta-analysis of child and adolescent outcomes. Aggress Violent Behav 2008;13:131-40.

9. Chan Y-C, Yeung JW-K. Children living with violence within the family and its sequel: A meta-analysis from 1995-2006. Aggress Violent Behav 2009;14:313-22.

10. Felitti VJ, Anda RF, Nordenberg D, et al. Relationship of childhood abuse and household dysfunction to many of the leading causes of death in adults. The Adverse Childhood Experiences (ACE) Study. Am J Prev Med 1998;14:245-58.

11. Lieberman AF, Weston DR, Pawl JH. Preventive intervention and outcome with anxiously attached dyads. Child Dev 1991;62:199-209.

12. Segal L, Guy S, Furber G. What is the current level of mental health service delivery and expenditure on infants, children, adolescents, and young people in Australia? Aust N Z J Psychiatry 2018;52:163-72.

13. Hooker L, Kaspiew R, Taft A. Domestic and family violence and parenting: Mixed method insights into impact and support needs: State of knowledge paper: Australia's National Research Organisation for Women's Safety (ANROWS) Landscapes, 2016.

14. Goldblatt H, Buchbinder E, Cohen R. Re-Experiencing Motherhood: Transformation of Relationships Between Formerly Abused Women and Their Children. Violence Against Women 2014;20:561-80.

15. Peled E, Gil IB. The mothering perceptions of women abused by their partner. Violence Against Women 2011;17:457-79.

16. Humphreys C, Mullender A, Thiara R, et al. 'Talking to My Mum': Developing Communication Between Mothers and Children in the Aftermath of Domestic Violence. Journal of Social Work 2006;6:53-63.

17. Heward-Belle S. Exploiting the 'good mother' as a tactic of coercive control. Affilia 2017;32:374-89. 
18. Moulding NT, Buchanan F, Wendt S. Untangling Self-Blame and Mother-Blame in Women's and Children's Perspectives on Maternal Protectiveness in Domestic Violence: Implications for Practice. Child Abuse Review 2015;24:249-60.

19. Hooker L, Samaraweera NY, Agius PA, et al. Intimate partner violence and the experience of early motherhood: a cross-sectional analysis of factors associated with a poor experience of motherhood. Midwifery 2016;34:88-94.

20. Lieberman AF, Padrón E, Van Horn P, et al. Angels in the nursery: the intergenerational transmission of benevolent parental influences. Infant Ment Health J 2005;26:504-20.

21. Carpenter GL, Stacks AM. Developmental effects of exposure to Intimate Partner Violence in early childhood: A review of the literature. Child Youth Serv Rev 2009;31:831-9.

22. Rizo CF, Macy RJ, Ermentrout DM, et al. A review of family interventions for intimate partner violence with a child focus or child component. Aggress Violent Behav 2011;16:144-66.

23. Howarth E, Moore THM, Welton NJ, et al. IMPRoving Outcomes for children exposed to domestic ViolencE (IMPROVE): an evidence synthesis. Public Health Res 2016;4:1-342.

24. Gillies D, Maiocchi L, Bhandari AP, et al. Psychological therapies for children and adolescents exposed to trauma. Cochrane Database Syst Rev 2016;10:CD012371.

25. Howarth E, Moore THM, Shaw ARG, et al. The effectiveness of targeted interventions for children exposed to domestic violence: Measuring success in ways that matter to children, parents and professionals. Child Abuse Review 2015;24:297-310.

26. Graham-Bermann SA, Lynch S, Banyard V, et al. Community-based intervention for children exposed to intimate partner violence: an efficacy trial. J Consult Clin Psychol 2007;75:199-209.

27. Lieberman AF, Van Horn P, Ippen CG. Toward evidence-based treatment: child-parent psychotherapy with preschoolers exposed to marital violence. J Am Acad Child Adolesc Psychiatry 2005;44:1241-8.

28. Letourneau N, Tryphonopoulos P, Giesbrecht G, et al. Narrative and meta-analytic review of interventions aiming to improve maternal-child attachment security. Infant Ment Health $J$ 2015;36:366-87.

29. Lieberman AF, Ghosh Ippen C, Van Horn P. Don't hit my mommy!: $A$ manual for Child-Parent Psychotherapy with young children exposed to violence and other trauma. Washington DC: ZERO TO THREE, 2015.

30. Lieberman AF, Ghosh Ippen C, VAN Horn P. Child-parent psychotherapy: 6-month follow-up of a randomized controlled trial. $J$ Am Acad Child Adolesc Psychiatry 2006;45:913-8.

31. Cicchetti D, Rogosch FA, Toth SL. Fostering secure attachment in infants in maltreating families through preventive interventions. Dev Psychopathol 2006;18:623-49.

32. Toth SL, Maughan A, Manly JT, et al. The relative efficacy of two interventions in altering maltreated preschool children's representational models: implications for attachment theory. Dev Psychopathol 2002;14:877-908.

33. Cicchetti D, Toth SL, Rogosch FA. The efficacy of toddler-parent psychotherapy to increase attachment security in offspring of depressed mothers. Attach Hum Dev 1999;1:34-66.

34. Cicchetti D, Rogosch FA, Toth SL. The efficacy of toddler-parent psychotherapy for fostering cognitive development in offspring of depressed mothers. J Abnorm Child Psychol 2000;28:135-48.

35. Toth SL, Rogosch FA, Manly JT, et al. The efficacy of toddler-parent psychotherapy to reorganize attachment in the young offspring of mothers with major depressive disorder: a randomized preventive trial. J Consult Clin Psychol 2006;74:1006-16.
36. World Health Organization. Responding to intimate partner violence and sexual violence against women: WHO clinical and policy guidelines. Geneva: World Health Organization, 2013.

37. David P, Schiff M. Learning from bottom-up dissemination: Importing an evidence-based trauma intervention for infants and young children to Israel. Eval Program Plann 2015;53:18-24.

38. Kenny M, Hassett A, Pae L. Exploring how parents make sense of change in parent-child psychotherapy. Journal of Infant, Child, and Adolescent Psychotherapy 2017;16:73-92.

39. Humphreys C, Healey L, Kirkwood D, et al. Children living with domestic violence: a differential response through multi-agency collaboration. Australian Social Work 2018;71:162-74.

40. Morris A, Toone E, Utter M, et al. Extending the reach: A timely and tailored response to children who experience family violence. Parity 2011;24:37.

41. Bunston W, Eyre K, Carlsson A, et al. Evaluating relational repair work with infants and mothers impacted by family violence. Aust $N Z$ J Criminol 2016;49:113-33.

42. Stover CS. Throw-Away Dads? Promoting Healthy Father-Child Attachment in Families Affected by Intimate Partner Violence. Zero to Three 2015;35:36-42.

43. May C, Finch T. Implementing, embedding, and integrating practices: an outline of normalization process theory. Sociology 2009;43:535-54

44. QSR International. NVivo qualitative data analysis software, 2013. Version 11.

45. Stata Statistical Software: Release 15. [program]. College Station, Texas, USA: StataCorp LLC, 2017.

46. Matias C, Scott S, O'Connor T. Coding of attachment-related parenting (CARP). London, UK: Institute of Psychiatry, Kings College, 2006.

47. Robling M, Bekkers MJ, Bell K, et al. Effectiveness of a nurse-led intensive home-visitation programme for first-time teenage mothers (Building Blocks): a pragmatic randomised controlled trial. Lancet 2016;387:146-55.

48. Lynch J. What can early interventions really acheive and how will we know? Family Matters 2017;99:15-22.

49. Derogatis LR, Unger R. Symptom checklist-90-revised. The corsini encyclopedia of psychology: John Wiley \& Sons, Inc, 2010.

50. Foa EB, Riggs DS, Dancu CV, et al. Reliability and validity of a brief instrument for assessing post-traumatic stress disorder. $J$ Trauma Stress 1993;6:459-73.

51. Ford-Gilboe M, Wathen CN, Varcoe C, et al. Development of a brief measure of intimate partner violence experiences: the Composite Abuse Scale (Revised)-Short Form (CASR-SF). BMJ Open 2016;6:e012824.

52. Goodman R. Psychometric properties of the strengths and difficulties questionnaire. J Am Acad Child Adolesc Psychiatry 2001;40:1337-45.

53. Scheeringa MS, Haslett N. The reliability and criterion validity of the Diagnostic Infant and Preschool Assessment: a new diagnostic instrument for young children. Child Psychiatry Hum Dev 2010;41:299-312.

54. Zubrick SR, Lucas N, Westrupp EM, et al. Parenting measures in the Longitudinal Study of Australian Children: Construct validity and measurement quality, Waves 1 to 4. Canberra: Department of Social Services, 2014

55. Luyten P, Mayes L, Sadler L, et al. The Parental Reflective Functioning Questionnaire-1 (PRFQ-1). Leuven, Belgium: University of Leuven, 2009. 